\title{
Bulk tank raw milk microbiota differs within and between farms: A moving goalpost challenging quality control
}

\author{
Siv B. Skeie, Monica Håland, Inga M. Thorsen, Judith Narvhus, and Davide Porcellato* \\ Faculty of Chemistry, Biotechnology and Food Science, Norwegian University of Life Sciences, PO Box 5003, N-1432 Ås, Norway
}

\section{ABSTRACT}

Microbial contamination of bovine raw milk often occurs at the farm. To acquire a deeper knowledge of the microbiota of farm tank milk, we studied milk from 45 farms situated in 2 geographical areas in Norway. Each farm was visited on 3 different occasions, with at least 2 wk between visits. We combined both bacterial cell counts and a sequence variant inference method of amplicon-based high-throughput sequencing to achieve a high-resolution overview of the microbiota in each sample. Compositional variation of the farm milk microbiota was shown in relation to the 2 areas, between the farms and between the sampling times. Despite the near constant level of bacteria enumerated in milk from each individual farm, the dominant microbiota differed significantly between the samplings. The predominant microbiota was dominated by spoilage genera, such as Pseudomonas and Bacillus, as well as the dairy fermentation genus Lactococcus and mastitis-causing organisms (Streptococcus). Analysis of the identified sequence variants within these genera showed that the populations of Pseudomonas and Lactococcus in milk had similar composition between the farms, but that Bacillus and, in particular, Streptococcus populations changed between collection days from the same farm and between farms and geographical areas. Furthermore, the levels and composition of Bacillus and Paenibacillus were different between the 2 geographical areas. The results presented here provide new insight into the farm milk microbiota and show that this microbiota is a dynamic community highly subject to variation.

Key words: raw milk microbiota, farm, sequence variant inference, bacterial cell count

Received November 2, 2017.

Accepted October 7, 2018.

*Corresponding author: davide.porcellato@nmbu.no

\section{INTRODUCTION}

Bovine raw milk has one of the most diverse microbial communities detected in food matrixes, and this diversity is one of the factors causing shifts in milk pricing, safety, and quality of both raw milk and dairy products (Quigley et al., 2013b). Although the dairy industry attempts to strictly control microbial quality throughout the milk processing lines, a large number of bacteria that can cause quality problems are constantly transported to the dairy plant with the raw milk. These bacteria include spoilage bacteria such as psychrotrophs, thermoduric and thermo-resistant bacteria, and pathogens, and these are regularly detected in milk during storage in silo tanks and in the dairy environment (Cleto et al., 2012; von Neubeck et al., 2015; Porcellato et al., 2018). Spore-forming microorganisms and thermoduric enzymes produced by psychrotrophs are not destroyed by pasteurization and represent common quality problems in milk and dairy products. Therefore, greater control of the initial microbial composition of the raw milk is of fundamental importance for the dairy industry in order to reduce or limit economical losses caused by quality problems.

Microbiological contamination of raw milk takes place mainly at the farm level, and bacteria may originate from a variety of sources, ranging from inside the udder to the milking and storage equipment. Milk is not sterile within the udder of healthy cows, as previously thought, and contains a wide range of microorganisms, including Ruminococcus, Bifidobacterium, and Peptostreptococcaceae, which are thought to enter the milk via the entero-mammary pathway (Young et al., 2015). In addition, contamination occurs from the teat surface microbiota and from the milking and milk handling equipment (Elmoslemany et al., 2010). The mastitic udder sheds large numbers of pathogenic microorganisms (including Streptococcus, Staphylococcus, and Escherichia) into the milk. Considering all this, the milk microbiota is understandably a complex community where many different species from diverse sources are present, and this becomes a challenge for the dairy 
industry, which is required to produce high-quality milk and milk products.

Many bacterial taxa have been detected in raw milk by both culture-dependent and culture-independent methods (reviewed by Quigley et al. 2013a). In recent studies performed with amplicon-based high-throughput sequencing (HTS), the number of different taxa detected in bovine raw milk samples was shown to be in the order of thousands (Hou et al., 2015; Kable et al., 2016; Doyle et al., 2017a). Despite this overall diversity, some bacteria groups were frequently found in studies using both culture-dependent and cultureindependent approaches. Of these, the most abundant were lactic acid bacteria, psychrotrophic bacteria, and spore-forming bacteria (Quigley et al. 2013b).

New methods have recently been applied to describe the composition of the raw milk microbiota (Kable et al., 2016; Doyle et al., 2017b; Rodrigues et al., 2017). These methods, based on culture-independent approaches and amplicon-based HTS, are able to successfully identify changes in microbiota associated with seasonal housing, farm management, and the transfer of milk from truck to milk-processing facilities. These techniques allow an in-depth analysis of the milk microbiota and are able to detect taxa not found using traditional cultivation methods (Quigley et al., 2011). However, the use of amplicon-based HTS suffers from bias because the values obtained are transformed to relative abundance. Therefore, the abundancy of taxa found cannot be converted to real values and, as they are dependent on each other, results for different samples cannot be readily quantitatively compared (Xia and Sun, 2017). Methods for absolute quantification have been implemented in some of these studies (by quantitative PCR), but these results were not directly linked to the abundance of several different taxa (Doyle et al., 2017a). New bioinformatics tools, with greater resolution than before, are being continuously developed to study microbial community data (Callahan et al., 2016). These new methods can be used to provide a better description of the diversity of the milk microbiota so that the dairy industry can act appropriately to improve microbiological quality of their milk and dairy products.

The objective of our study was to obtain an overview of the microbial composition and diversity of raw milk (1) between different collection days at the same farm, (2) between farms, and (3) between geographical regions. The microbiota was studied by applying a novel approach of combining the culture-independent information from the bacterial counts with amplicon-based HTS-sequencing. Further, we examined the application of sequence variant (SV) inference as an alternative method to OTU clustering to obtain a better resolution of the microbiota determination.

\section{MATERIALS AND METHODS}

\section{Study Design, Milk Samples, and Sampling}

Forty-five farms, distributed within 2 geographical areas in Norway (20 farms in area A and 25 farms in area B), were selected for this experiment. The farms from each area deliver bovine raw milk to 2 regional dairy plants, which are situated $110 \mathrm{~km}$ apart in the southern part of Norway, where climate conditions and elevation are similar. Each farm was visited on 3 occasions between January and March 2017, with 2 wk between each sampling for farms in area $\mathrm{A}$ and $3 \mathrm{wk}$ between each sampling in area B. In Norway, farm milk is usually collected after 2 or $3 \mathrm{~d}$ of storage at the farm. In this study, the milk was collected from farms where collection of the milk is performed only every $3 \mathrm{~d}$. The milk was collected on the same weekday and was a mix of 6 consecutive milking (evening on d 1, morning and evening on $\mathrm{d} 2$ and 3 , and morning of the collection day). The farms were categorized based on the type of milking system (free roaming with parlor milking system, free roaming with automatic milking system, and stall with pipeline milking system; Supplemental Table S1, https://doi.org/10.3168/jds.2017-14083). At each sampling, raw milk was collected during the transfer from the bulk farm tank to the tanker truck. The milk was collected in a sterile $50-\mathrm{mL}$ tube using the sampling outlet of the truck and stored at $4^{\circ} \mathrm{C}$ during transportation. Immediately after arrival at the dairy plant, milk from each sample was removed for Bactocount analysis (Bentley Instrument Inc., Chaska, MN), and the remainder was transported at $4^{\circ} \mathrm{C}$ (within $2 \mathrm{~h}$ ) to the Faculty of Chemistry, Biotechnology and Food Science laboratory, Norwegian University of Life Sciences, for DNA extraction on the same afternoon, evening, or early the following morning.

\section{Bacterial Level in Milk Samples}

Flow cytometric counting of the bacteria in the milk samples was performed using the Bactocount IBCm instrument (Bentley Instrument Inc.). Milk was analyzed for total bacterial count (TBC) immediately after arrival of the truck at the dairy plant. One milliliter of milk was taken from the collection tube and the analysis was performed according to manufacturer's instructions (Bentley Instrument Inc.; ftp://ftp.bentleyinstruments .com/Bactocount-Manual.pdf). 


\section{DNA Extraction from Bacteria in Milk Samples}

A bacterial pellet was prepared from $40 \mathrm{~mL}$ of each milk sample. The milk was centrifuged for $10 \mathrm{~min}$ at $8,000 \times g$ at $4^{\circ} \mathrm{C}$ and the pellet was resuspended and washed twice with $1 \mathrm{~mL}$ of $2 \%$ sodium citrate water (wt/vol). The pellet was then resuspended in $200 \mu \mathrm{L}$

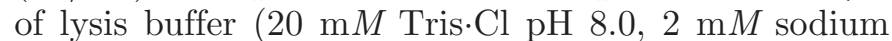
EDTA, 1.2\% Triton X-100, Sigma-Aldrich, St. Louis, $\mathrm{MO}$ ) and transferred to a Fastprep tube (MP Biomedicals, Santa Ana, CA) containing $0.5 \mathrm{~g}$ of acid-washed glass beads (Sigma-Aldrich). The tubes were subjected to 3 rounds of bead-beating in a Fastprep-96 Instrument (MP Biomedicals) for $50 \mathrm{~s}$ at $1600 \mathrm{rpm}$ and cooled on ice for $1 \mathrm{~min}$ between bead-beating rounds. The tubes were then centrifuged for $1 \mathrm{~min}$ at 16,000 $\times g$ at room temperature and $50 \mu \mathrm{L}$ of supernatant was used for DNA extraction. The DNA extraction was performed using the Mag Midi kit (LGC, Luckenwalde, Germany) according to manufacturer's instructions (https://www .lgcgroup.com/LGCGroup/media/PDFs/Products/ Extraction/Kits\%20inserts/mag-midi.pdf); DNA was stored at $-20^{\circ} \mathrm{C}$.

\section{S rRNA Marker Gene Sequencing and Bioinformatics}

The DNA extracted from the milk samples was amplified using the method described by Porcellato and Skeie (2016) with minor changes. Briefly, $2 \mu \mathrm{L}$ of DNA was added to the $\mathrm{PCR}$ reactions mix containing $1 \times \mathrm{HF}$ Buffer (Biorad, Hercules, CA), $0.5 \mathrm{mg} / \mathrm{mL}$ of BSA, 0.2 $\mathrm{m} M$ of each primer, $200 \mathrm{mM}$ of each deoxynucleotide triphosphate, and $0.02 \mathrm{U} / \mathrm{mL}$ of iProofTM taq polymerase (Biorad). The PCR amplification conditions were similar to those previously reported (Porcellato and Skeie, 2016). The PCR product was purified using 0.6× Agencourt AMPure XP beads (Beckman Coulter Inc., Brea, CA) according to the manufacturer's instructions (https://www.beckmancoulter.com/wsrportal/ techdocs?docname=B37419). Five microliters of the purified PCR product was used as a template for the second PCR using primers with unique 8-bp barcodes. The PCR reaction was similar to the previous PCR and the amplification was performed as follows. Initial denaturation at $98^{\circ} \mathrm{C}$ for $30 \mathrm{~s}$ and 10 cycles of denaturation for $15 \mathrm{~s}$, annealing for $30 \mathrm{~s}$ at $55^{\circ} \mathrm{C}$, and elongation for $20 \mathrm{~s}$ at $72^{\circ} \mathrm{C}$. Final elongation was performed for 5 min at $72^{\circ} \mathrm{C}$. The PCR product was then purified and normalized using SequalPrep Normalization Plate Kit (Thermo Fisher Scientific, Waltham, MA). The purified library was quantified using the Kapa library quantification kit (Kapa Biosystems, Wilmington, MA) and sequenced on an Illumina Miseq platform (Il- lumina Inc., San Diego, CA) using the Miseq Reagent Kit v3 (Illumina Inc.). Sequences were deposited at the European Nucleotide Archive with accession number PRJEB24669. Raw files were merged and qualityfiltered using Usearch 8.0 with Max E-value of 0.5 (Edgar, 2010). Reads were assigned to the different SV using the R package "dada2" (Callahan et al., 2016). Assignation of taxon for each SV was performed after training the classifier using the RDP training set 14 and the function "assignTaxonomy" from the $\mathrm{R}$ package "dada2" (Callahan et al., 2016).

\section{Statistical Analysis}

All the statistical analysis was performed using $\mathrm{R}$ version 3.3.3 ( $\mathrm{R}$ Core Team, 2017). The TBC was calculated per milliliter of milk and then transformed to $\log$ TBC per milliliter. The TBC in milk samples collected from different farm types and milking systems were compared using the Kruskal-Wallis test. The type of farm and the milking system were used as a single independent variable with 3 levels: free roaming with parlor milking system, free roaming with automatic milking system, and stall with pipeline milking system. The SV table was normalized using the cumulative-sum scaling method using the R package "metagenomeSeq" (Paulson et al., 2013). Permutational analysis using the same dissimilarity matrixes was used to test differences in the composition of the community between groups of samples (number of permutations $=999$ ). Principal coordinate analysis was chosen as ordination method using 3 distance matrixes: weighted and unweighted UniFrac dissimilarities (Lozupone and Knight, 2005) and Bray-Curtis. Samples with less than 2,000 sequences $(n=7)$ were not considered for statistical analysis, but were included in the relative and absolute abundance analysis. The relative abundance of each taxon was transformed to absolute value $(\log \mathrm{TBC} / \mathrm{mL})$ after computing the percentage of that taxon according to the total levels obtained from the Bactocount analysis for each sample. This was calculated by considering $\mathrm{q}$ copy of $16 \mathrm{~S}$ rRNA per TBC. Statistical analysis of the genus-associated SV was performed using the BrayCurtis distance matrix and permutational multivariate ANOVA between groups of samples; a $P$-value $<0.05$ was considered significant. The nonparametric MannWhitney U test was used to compare the abundance of the most abundant genera (expressed as $\log \mathrm{TBC} / \mathrm{mL}$ ) between the 2 geographical areas. One consideration during the analysis of the SV results was that the number of SV is correlated with the phylogenetic variation of the amplified 16S fragment (in this study the V3 and $\mathrm{V} 4$ region of the $16 \mathrm{~S}$ rRNA) of the genus considered; 
thus, the results do not take into consideration the number of $16 \mathrm{~S}$ copies present in the bacterial genome.

\section{RESULTS}

The TBC in the 135 milk samples ranged from log 3.6 to $\log 5.28 \mathrm{TBC} / \mathrm{mL}$, with a mean value of $4.27 \mathrm{TBC} /$ $\mathrm{mL}$ and median of $4.25 \mathrm{TBC} / \mathrm{mL}$. Only 2 samples out of 135 showed a log TBC per milliliter greater than 5 . Overall, the TBC was similar between the 3 samplings from each single farm with few exceptions, such as farm 34 , where the bacterial count of sample 2 was $1 \mathrm{log}$ $\mathrm{TBC} / \mathrm{mL}$ greater than the other 2 samples (Figure 1 ). Milk from parlor farms with automatic milking systems showed a greater bacterial count than the other 2 types of farm with different milking system. The overall TBC was significantly different $(P<0.05)$ between the 3 types of farms, and distribution of the TBC grouped by type of farm is shown in Supplemental Figure S1 (https://doi.org/10.3168/jds.2017-14083).

From the 16S rRNA sequencing, an average of 11,667 (median $=8,515)$ sequences per sample was obtained. The sequences were distributed in 5,490 SV, representing 105 taxonomical families and 334 genera. Microbial communities of the raw milk samples were grouped according to the geographical area of collection and the sampling times using principal coordinate analysis (Figure 2). Furthermore, permutational ANOVA showed these grouping to be diverse (Adonis $P<0.05$ ) with respect to geographical area and sampling time (Table 1). The microbiota in the milk samples from individual farms showed poor homogeneity between the collections days (Figure 1). The large distribution of the homogeneity between collection days at the same farm indicates that the microbiota composition differs between sampling, for example on farm 1, 8, and 28 .

The most abundant genera we detected were Pseudomonas, Lactococcus, Bacillus, and Streptococcus, accounting for $26.6,12.0,11.0$ and $6.4 \%$ of the total sequences, respectively; these values were converted to give an average abundance per sample of 3.6, 3.2, 2.6, and $2.7 \log \mathrm{TBC} / \mathrm{mL}$, respectively. Whereas Lactococcus and Streptococcus levels did not differ between the 2 different areas $(P>0.05)$, the levels of Pseudomonas and Bacillus were significantly different between the 2 geographical areas (Figure 3). The levels of other genera, such as Staphylococcus, Paenibacillus, Psychrobacter, Chryseobacterium, Aerococcus, and Rhizobium, also differed significantly between the 2 areas (Figure 3 ).

The milk microbiota from the same farms was not similar at the 3 sampling times. The dominant genera in milk samples collected from the same farms varied

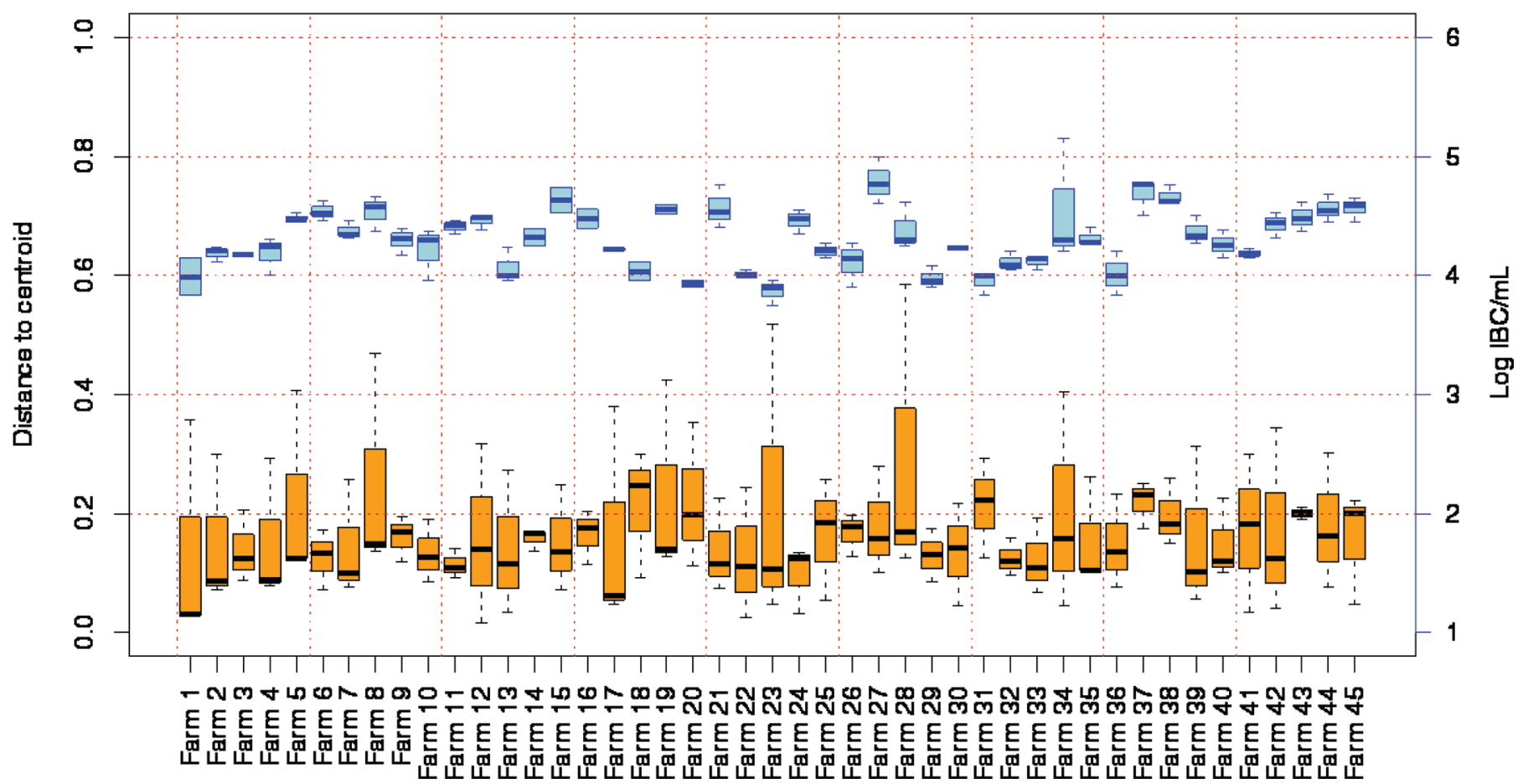

Figure 1. Average individual bacterial counts (IBC) from bulk tank milk collected from each farm (blue) and the homogeneity of group dispersion (beta diversity: orange) at each farm obtained from the weighted Unifrac dissimilarity matrix (Lozupone and Knight, 2005). Milk samples from farm 1 to 20 were collected from area A, and from farms 21 to 45 from area B. The top and bottom of the box indicate the third and first quartile, whereas the middle line indicates the median. The upper and lower whiskers indicate the greatest and least value excluding outliers, respectively. 


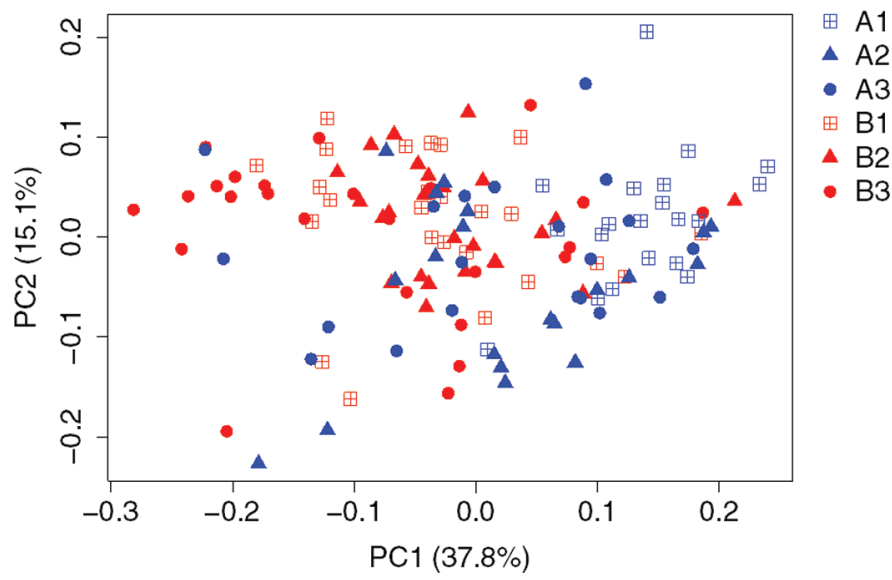

Figure 2. Principal coordinate (PC) analysis plot obtained from the weighted Unifrac distance matrix (Lozupone and Knight, 2005) for the microbiota of 135 milk samples. A1 = area A, sampling 1; A2 = area $\mathrm{A}$, sampling $2 ; \mathrm{A} 3=$ area $\mathrm{A}$, sampling $3 ; \mathrm{B} 1$ : area $\mathrm{B}$, sampling 1 ; $\mathrm{B} 2=$ area $\mathrm{B}$, sampling $2 ; \mathrm{B} 3=$ area $\mathrm{B}$, sampling 3.

between the collection days (Figure 4). For example, the genera Bacillus (in farm 1, 2, 9), Acinetobacter (in farm 19, 21), Paenibacillus (in farm 23), Staphylococcus (in farm 44), and Chryseobacterium (in farm 28) varied in relative abundance between the 3 sampling days. In addition, the genus Bacillus was present in greater abundance from most of the farms in collection area A compared with Paenibacillus, which showed greater abundance from farms in area B (Figure 3). On one of the collection days at farm 34, a marked increase in the total bacteria level was found to be due to a specific increase of Streptococcus (Figure 4).

Of the 5,490 SV, Corynebacterium was the genus with the highest number of SV detected $(\mathrm{n}=278)$, although it only represented $2.38 \%$ of the total number of reads. Of the 4 most abundant taxa, the genus Pseudomonas was divided into $132 \mathrm{SV}$ and Lactococcus into 206. In addition, Pseudomonas showed the highest number of SV per sample $($ mean $=10.4$, median $=10)$. The distribution of the SV in each sample showed that some of the dominant Pseudomonas and Lactococcus SV could be detected in all the samples with few exceptions, such as farms $7,8,9,15,18$, and 20 for Pseudomonas and farms 3, 4, 11, 14, 16, 17, and 21 for Lactococcus (Figure 5). Permutational ANOVA indicated that the Pseudomonas and Lactococcus populations were significantly different according to collection area, sampling time, and the type of housing or milking systems.

Bacillus was detected in all the samples from area $\mathrm{A}$ and also from $75 \%$ of the samples from area B (Figure 6 ), but in lower numbers (Figure 3). On the other hand, Paenibacillus was detected only in 32 and $68 \%$ of the samples from area A and B, respectively. Analysis of the SV for these 2 genera clearly showed the difference in the presence and abundance of these genera in the milk samples. The genus Bacillus was divided in $56 \mathrm{SV}$, whereas $50 \mathrm{SV}$ were detected within the genus Paenibacillus. The genus Bacillus detected in milk from

Table 1. Sequence variants description of the 15 most abundant bacterial genera found in the raw milk from 45 farms

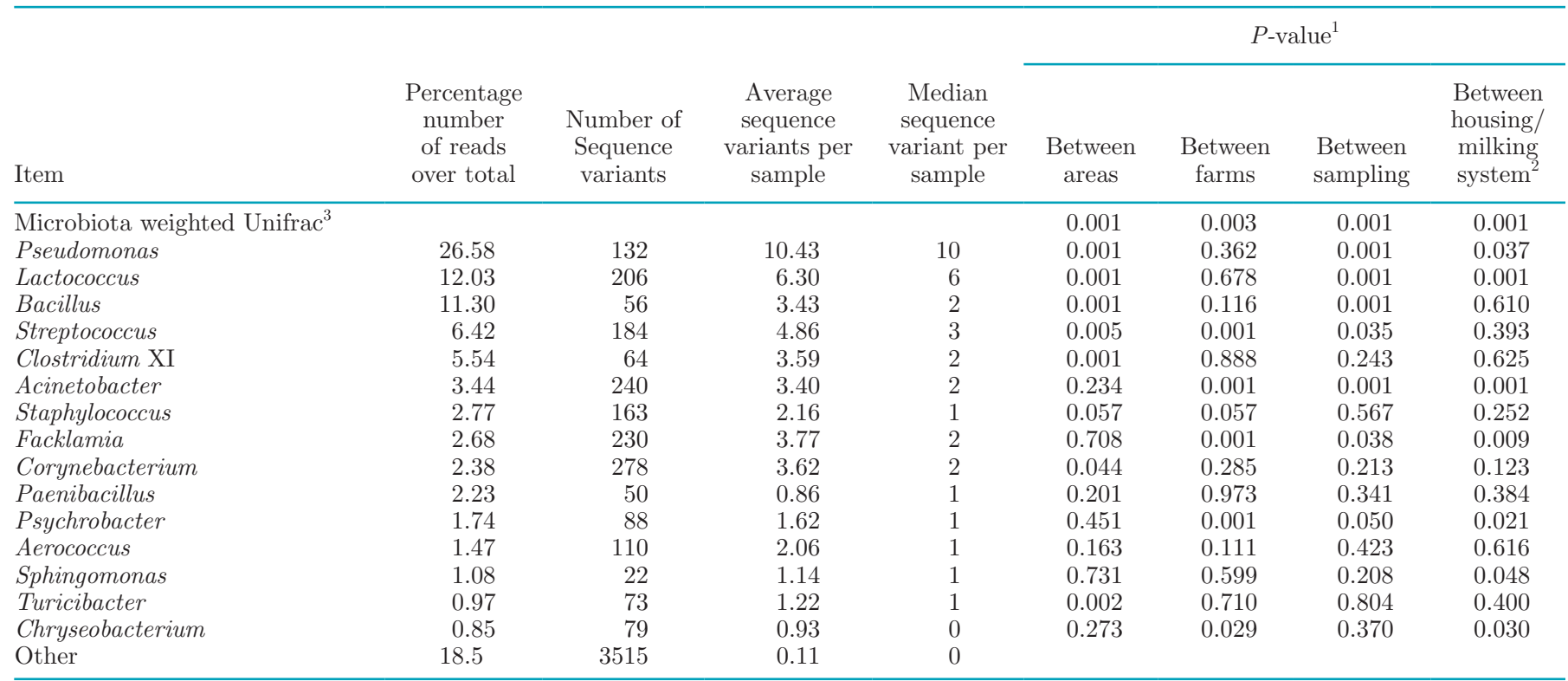

${ }^{1} P$-value obtained from the permutational multivariate ANOVA (R function Adonis from R package "Vegan"; R Core Team, 2017).

${ }^{2}$ Type of housing/milking system: free roaming with parlor milking system, free roaming with automatic milking system, and stall with pipeline milking system.

${ }^{3}$ Unifrac distance matrix (Lozupone and Knight, 2005). 

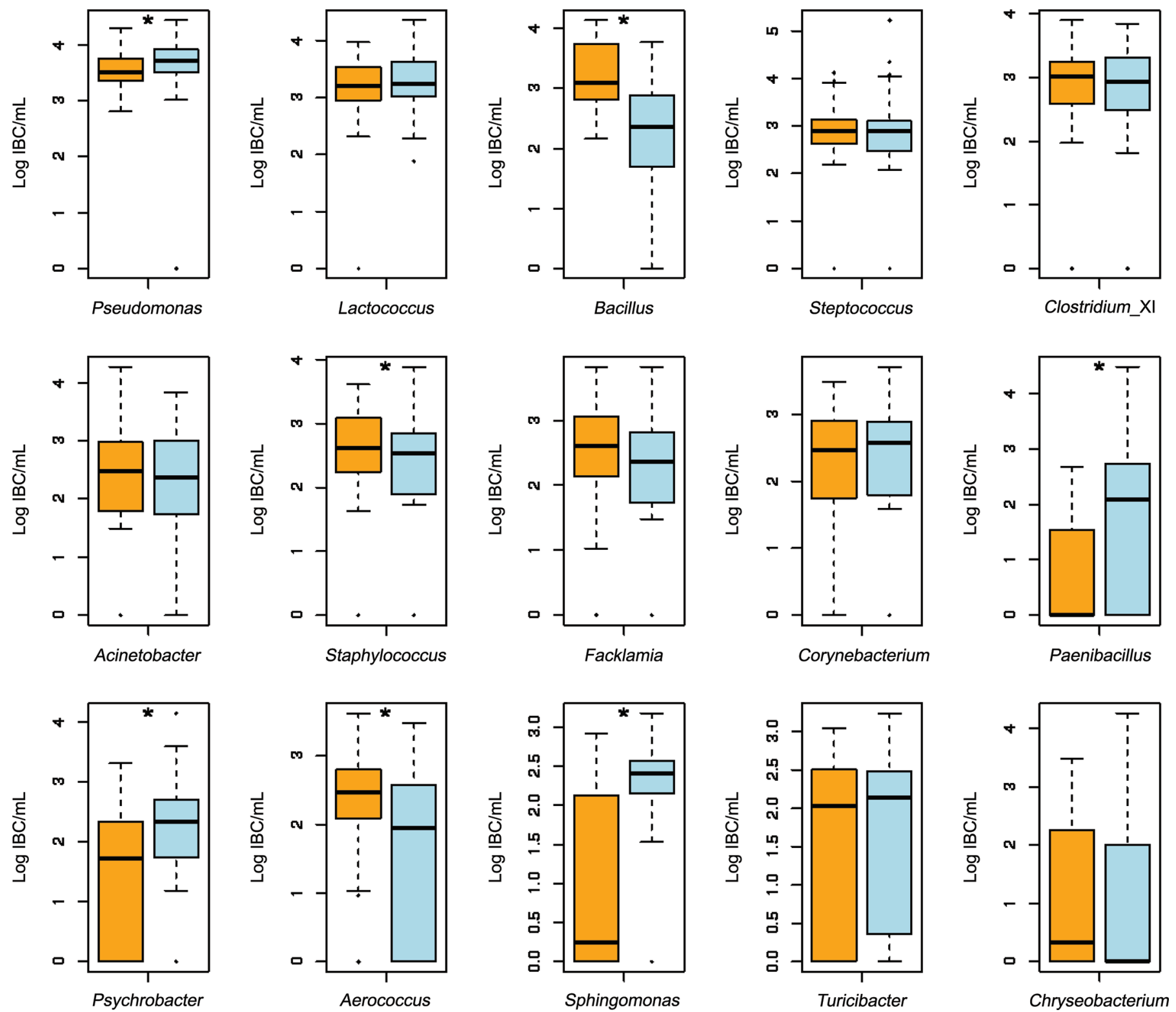

Figure 3. Distribution [log individual bacterial counts (IBC)/mL] of the 15 most represented genera of bacteria detected in 135 samples of raw milk from farm bulk tanks divided in the 2 geographical areas in Norway. Orange $=$ area A. Blue $=$ area B. The top and bottom of the box indicate the third and first quartile, whereas the middle line indicates the median. The upper and lower whiskers indicate the greatest and least value excluding outliers, respectively. *Significant differences $(P<0.05)$ between the 2 areas.

area A (farms 1 to 20) showed similar SV distribution (with few exceptions) between the farms, whereas in milk from farms in area B (farms from 21 to 45) the SV types were diverse both between farms and with respect to sampling days. For Paenibacillus, the SV distribution was also different between milk collected in the 2 areas, between farms, and between collection days.

The composition of the Streptococcus SV varied with respect to farm, sampling day, and area (Figure 7). The sample with extremely large numbers of Streptococcus (collection d 2 from farm 34) contained a mix of 13 different Streptococcus SV (Figure 7). Interestingly, 9 of the SV detected in the second day of collection from that farm were also detected in the last day of collection, whereas none of the SV from the first day of collection were detected in the second day of collection.

The type of housing or milking system had a significant effect on the total microbial composition at genus level. Both Lactococcus and Pseudomonas SV compositions were significantly influenced by the housing types, as were Acinetobacter, Facklamia, and Psychrobacter ( $P$ $<0.05)$. 


\section{DISCUSSION}

We demonstrated that the microbial composition of raw milk in farm milk tanks differs (1) between collection days for the same farm, (2) between farms, and (3) between regions. This was investigated using a novel combination of culture-independent bacterial count and next-generation sequencing, which allowed the quantitative evaluation of the composition of the milk microbiota.
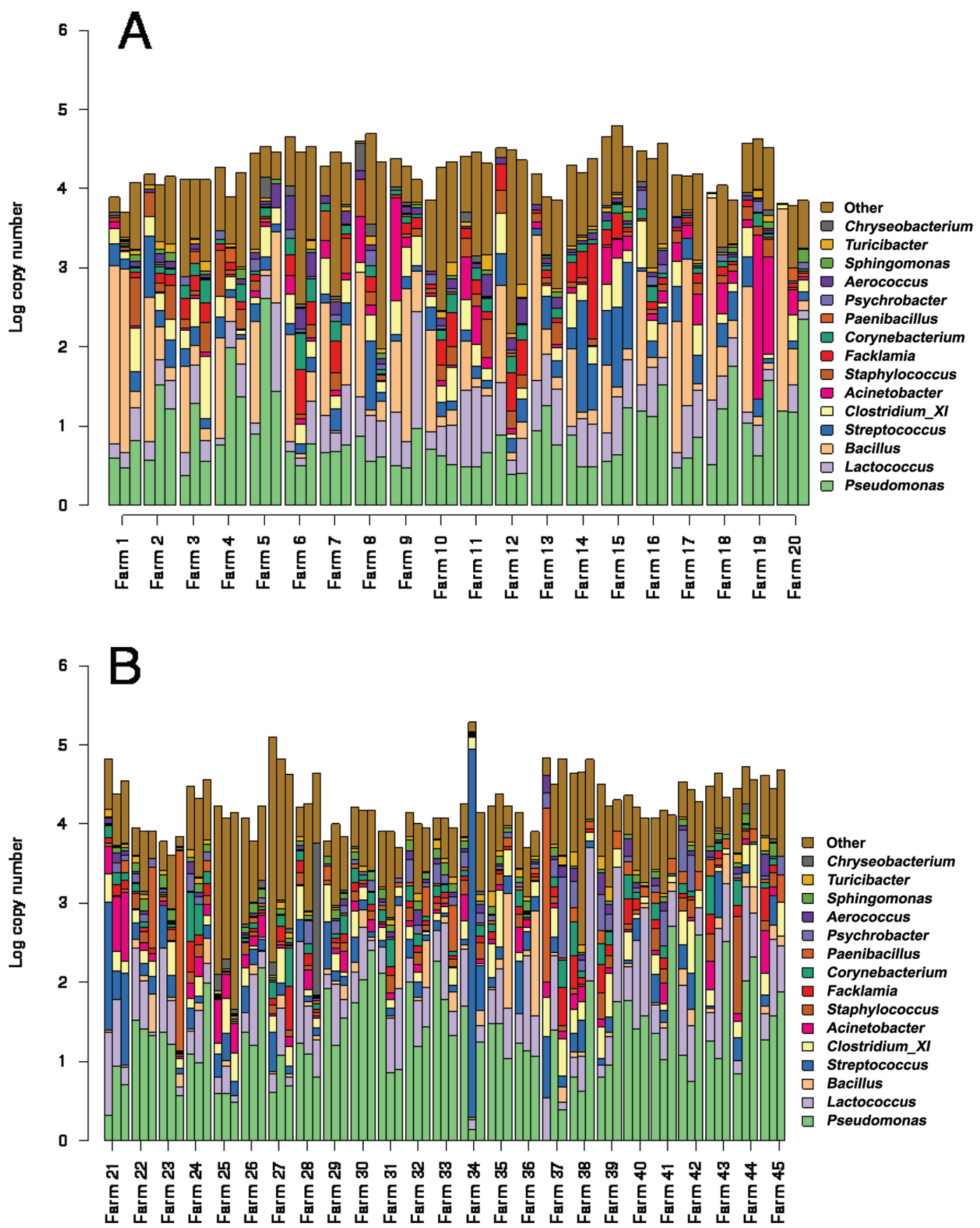

Figure 4. Distribution of the 15 most abundant sequence variants of bacterial genera detected in raw milk samples. Each bar represents a sample of milk and each farm was sampled 3 times. The height of each bar indicates the absolute level of bacteria (log copy number) obtained by normalizing genera relative abundances (in \%) against the absolute bacterial level. (A) Farm 1 to 20 collected in area A; (B) farm 21 to 45 collected in area B. 

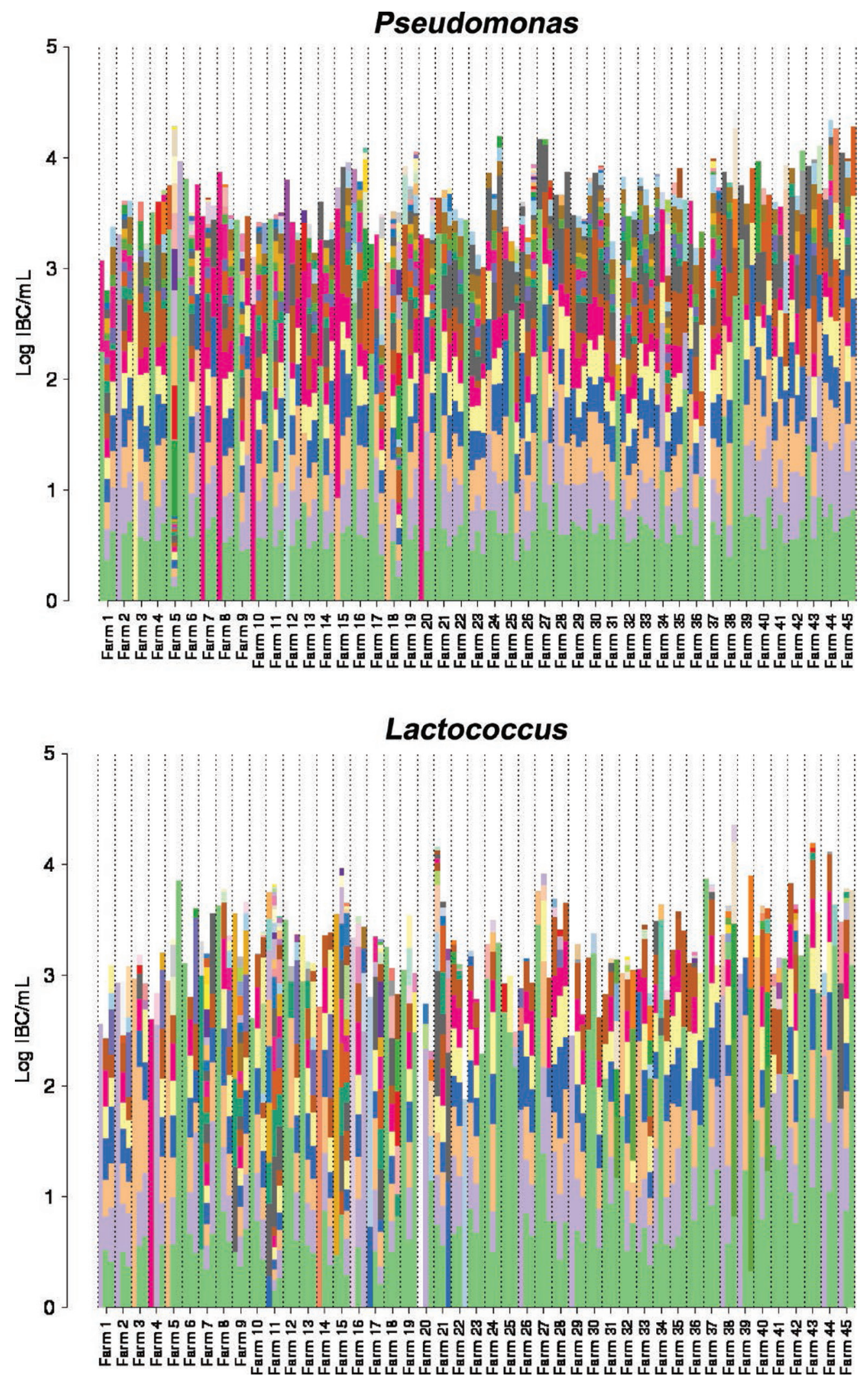

Figure 5. Distribution of the sequence variants for Pseudomonas and Lactococcus detected in the milk samples from 45 farms. Each color indicates a different sequence variant expressed as relative abundances of the height of the bar. The height of the bar indicates the absolute level of the genus indicated calculated over the total bacterial level [log individual bacterial counts (IBC)/mL]. 


\section{Bacillus}

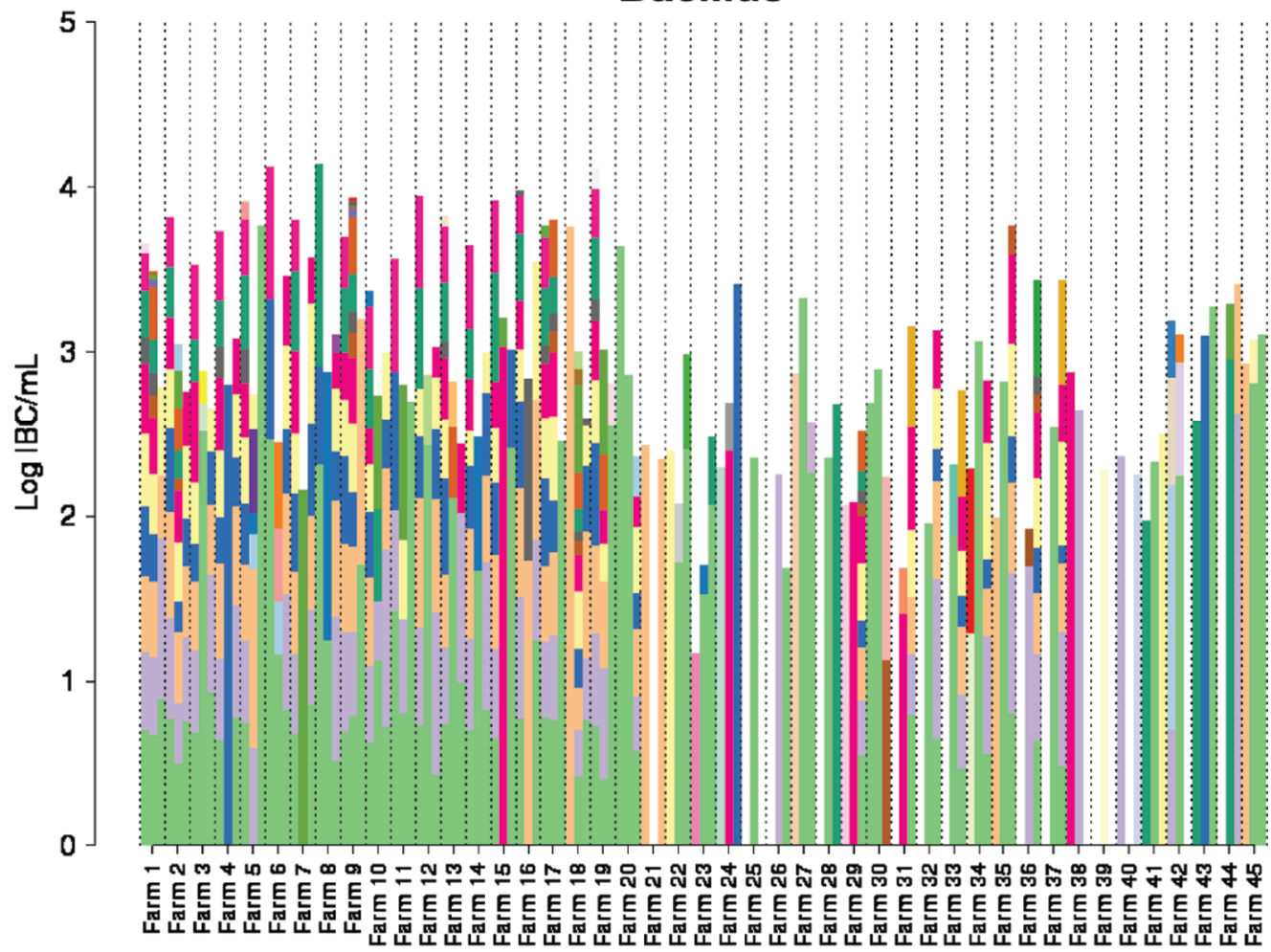

Paenibacillus

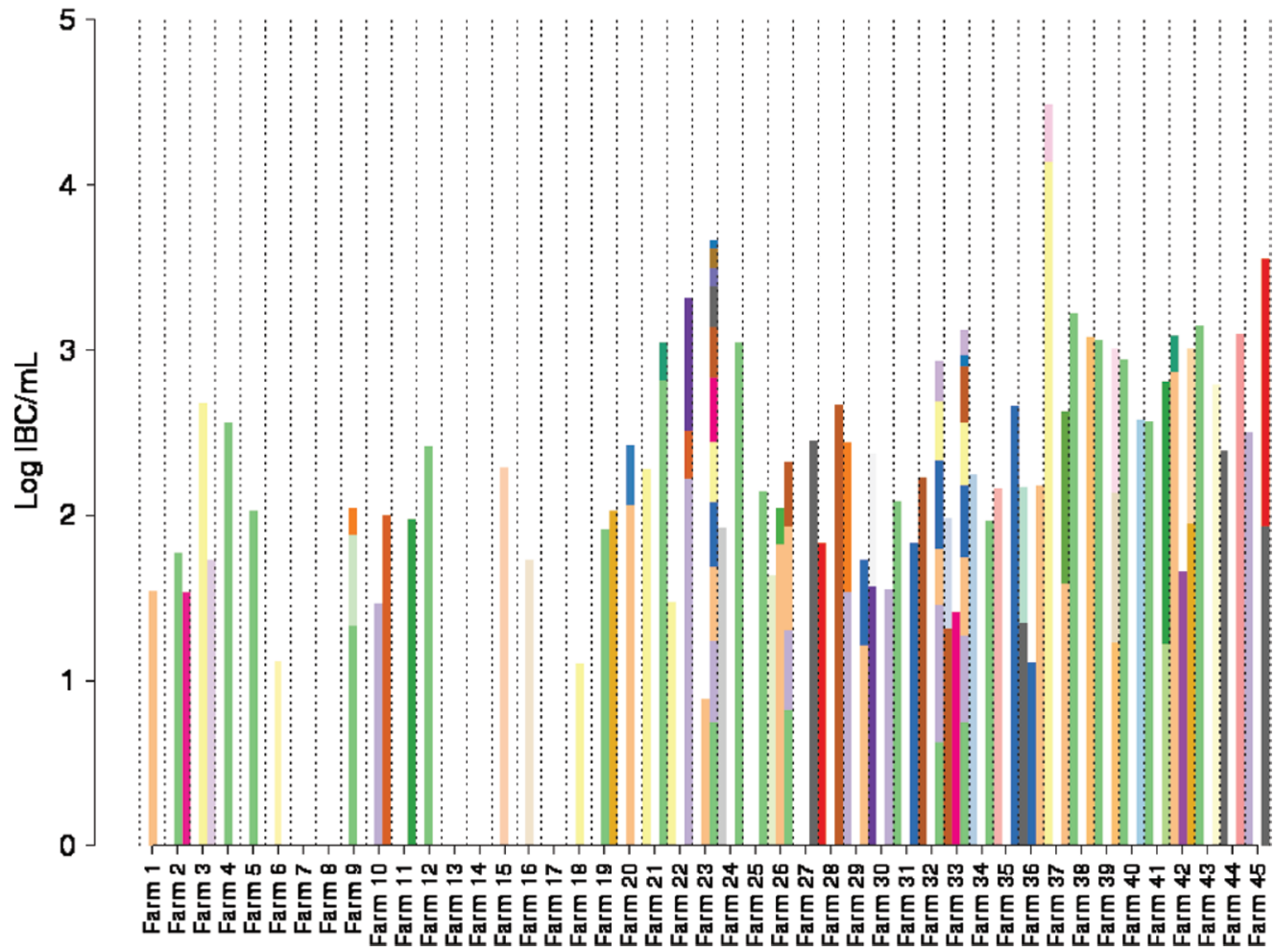

Figure 6. Distribution of the sequence variants for Bacillus and Paenibacillus detected in the milk samples from 45 farms. Each color indicates a different sequence variant expressed as relative abundances of the height of the bar. The height of the bar indicates the absolute level of the genus indicated calculated over the total bacterial level [log individual bacterial counts (IBC) $/ \mathrm{mL}$ ]. 


\section{Tank Milk Microbiota Between Collection Days}

Although the TBC was found to be similar in the 3 milk samples from individual farms, the microbial composition changed significantly between the collection days. This was detected for several farms, where an increased abundance of 1 or more genera were detected in 1 sample out of 3 from the same farm. This indicates that the composition varied randomly among samples from the same farms and, thus, removed the possibility of bias caused by sampling procedure. The TBC was performed the moment the milking tanker arrived at the dairy plant, whereas DNA extraction was performed the same day or the next morning after transportation to the university facility. Moreover, the milk was stored at $4^{\circ} \mathrm{C}$ and the same procedure was used for all samples from the same farms.

A shift in microbial composition between collection days at the same farm occurred over a period of 2 to 3 wk. Several factors, such as changes in feed quality, farm environment, weather conditions, and animal health, might have contributed to these changes (Hernandez-Sanabria et al., 2010; Vacheyrou et al., 2011; Quigley et al., 2013b; Oikonomou et al., 2014). However, we did not record such data from the farms, and the correlation between the microbial changes and these factors could not be directly identified. In farm 34 , for example, the second collection day showed an increase of the taxa Streptococcus and an increase in TBC, which might denote the presence of a significant mastitis problem in the herd at that time (Zadoks et al., 2004). Further, SV analysis of samples from farm 34 showed that the second collection day did not contain the same SV found in the first day of collection. The various streptococci might have been present but not detected, due to the detection limit of the method, on the first day, or new streptococci might have been introduced in the milk between collection days (e.g., new

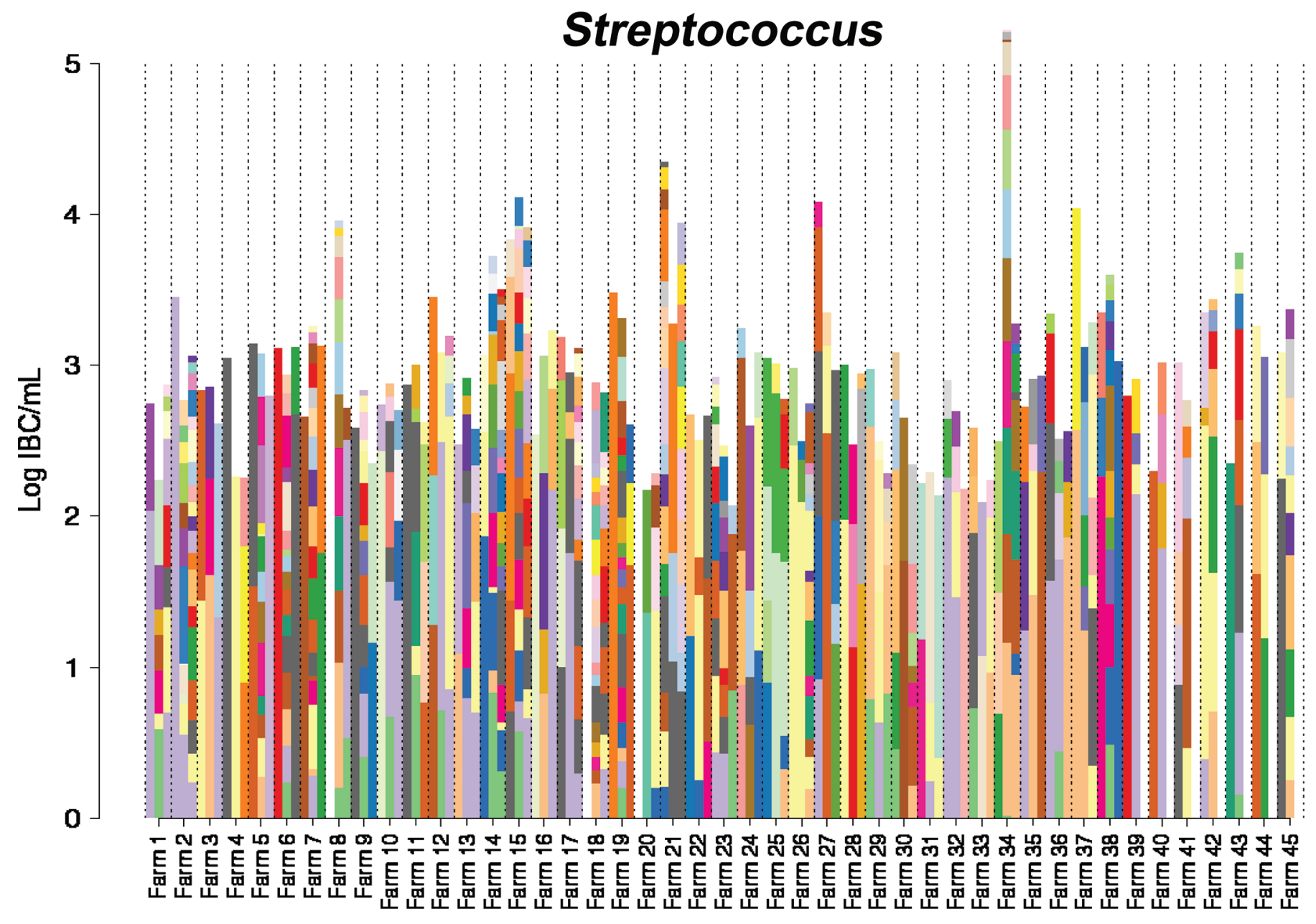

Figure 7. Distribution of the Streptococcus sequence variants detected in milk samples from 45 farms. Each color indicates a different sequence variant expressed as relative abundances of the height of the bar. The height of the bar indicates the absolute level of the genus indicated calculated over the total bacterial level [log individual bacterial counts (IBC)/mL]. 
cows might have been added to the herd). This suggests that the Streptococcus population is dynamic and continuously varied in composition between samplings at the same farm. We confirmed this by SV analysis of this genus, where the composition was highly diverse with respect to collection day, individual farm, and geographical location. In a previous study performed in Norway, mastitis-causing Streptococcus were identified in $4 \%$ of cows, independent of their udder health status (Reksen et al., 2006). In this present study, all the milk samples contained at least 1 Streptococcus SV, but identification at species level could not be inferred due to the short fragments sequenced. Streptococcus is a heterogeneous genus and includes several species found in the farm environment, such as on the teat skin (Braem et al., 2012) and dairy equipment (Marchand et al., 2012; Delgado et al., 2013). Some species are responsible for bovine mastitis (Waage et al., 1999; Osterås et al., 2006; Tenhagen et al., 2006).

Changes in milk microbiota between samplings have also been attributed to seasonal changes or weather conditions, such as temperature and humidity ( $\mathrm{Li}$ et al., 2018). We collected the milk samples during the same season (winter), which might have affected the level of some psychrotrophic bacteria. In particular, Pseudomonas, which was the most abundant genus we detected, have been previously correlated with winter milk (Vithanage et al., 2016; Porcellato et al., 2018). The level of Pseudomonas could also be explained by the 3 -d storage period of the milk at the farm before collection. The storage of milk for up to $3 \mathrm{~d}$ is a usual practice in the Norwegian dairy industry, and milk is kept at refrigerated temperature before being collected and processed. The low storage temperature encourages the growth of psychrotrophic bacteria, such as Pseudomonas and Acinetobacter (Vithanage et al., 2016).

\section{Tank Milk Microbiota Between Farms}

Both TBC and microbial composition were significantly different between milk samples collected at different farms, which is in accordance with findings in previous studies (Bonizzi et al., 2009; Feligini et al., 2015). This indicates that each farm can be considered as a particular niche containing a dynamic microbiota. On-farm management practices and milking systems were shown to have a great effect on the TBC and bacterial composition of milk (Elmoslemany et al., 2010; Doyle et al., 2017b). Overall, the TBC levels we detected were comparable to previous studies performed in farm milk samples from Norway and from other countries (Elmoslemany et al., 2010; De Jonghe et al., 2011; Fricker et al., 2011). The type of housing and milking system can also explain some of the differences in TBC in milk from different farms. Milk from farms with automatic milking systems showed higher TBC compared with the other 2 types of milking systems. This might be attributable to inferior washing of the teat, the lack of a drying step before milking, and challenges with equipment cleaning in the automatic milking system (Jago et al., 2006; Jacobs and Siegford, 2012).

Although significant differences exist between the farms, the 2 most abundant genera (Pseudomonas and Lactococcus) were detected in all milk samples (with few exceptions). Previously, Pseudomonas and Lactococcus were positively linked to milk from cows on pasture (Doyle et al., 2017b). However, our samples were collected from cows housed indoor, but these 2 genera represented over one-third of the total microbiota present in the milk. The great abundance of Pseudomonas in our samples might reflect the seasonal and storage conditions (as described above), whereas great abundance of Lactococcus was not surprising, as this genus has been described by other authors as one of the most abundant in raw milk samples (Quigley et al., 2013a; von Neubeck et al., 2015; Porcellato et al., 2018). In addition, the Lactococcus population isolated from raw milk samples has been previously shown to be dominated by psychrotolerant strains (von Neubeck et al., 2015). The composition of psychrotrophic and psychrotolerant microbiota affects the milk quality, in particular when low-temperature storage for several days is used before processing. In our study, both Lactococcus and Pseudomonas showed a great intragenus diversity (number of SV detected per sample), which might indicate multiple source of bacterial contamination in milk; this affects the complexity of the raw milk microbiota.

Another aspect that might have influenced the diversity between milk samples collected from different farms is the individual cow microbiome and the farm size. The average farm herd size in Norway is $27 \mathrm{cow} /$ farms (Statistics Norway, 2017), and in our study the milk was collected from large and small farms (amount of milk delivered after $3 \mathrm{~d}>4,000 \mathrm{~L}$ and $<1,000 \mathrm{~L}$, respectively). The first source of bacterial contamination in milk is the cow (udder and teat microbiome), and the number of cows in each farm might affect the diversity of taxa and SV detected in our study. For example, the diversity of Streptococcus could reflect the herd size, where farms with more cows could have a greater microbiota diversity. Here, the Streptococcus SV distribution was influenced by the farm and collection day, which indicates that the source of Streptococcus could be linked to the size of the herd. However, sampling from each individual cow is needed to evaluate the importance of each cow and farm size on the milk microbiota. 


\section{Tank Milk Microbiota in the 2 Sampling Areas}

We showed significant variation in milk microbiota related to the 2 geographical areas. Whereas the 2 areas were not different with regard to climate conditions and elevation, difference in farming practices, feed suppliers, and other sources of contamination could explain this variation (Mallet et al., 2012). Interestingly, 7 of the 15 most abundant genera were significantly different in level between the 2 areas, including Bacillus and Paenibacillus. Bacillus was more abundant in milk from area A and Paenibacillus was more abundant in milk samples from area B. These 2 bacterial genera are the predominant aerobic endospore-forming taxa detected in milk samples and are of concern to the dairy industry for their ability to survive pasteurization and cause subsequent spoilage in stored pasteurized milk (Coorevits et al., 2008; Ivy et al., 2012; Masiello et al., 2014). Several factors have been identified that influence the levels of aerobic endospore-forming bacteria in milk, such as bedding materials and feed (Vaerewijck et al., 2001; Magnusson et al., 2007), season variation (Bartoszewicz et al., 2008), and contamination from equipment (Salustiano et al., 2009). These factors could explain some of the differences between the 2 geographical areas of collection and can be assumed from the composition of the SV. Bacillus SV clearly showed a consistent presence of some dominant SV between collection days and farms in most milk samples from area A, whereas in milk samples from area B the Bacillus SV were different both within and between farms. Similar to Bacillus SV from area B, the Paenibacillus SV identified in all milk samples from area B were different between collection days and farms. Paenibacillus was detected in only $32 \%$ of the milk samples from area A as compared with $68 \%$ in milk from farms in area B.

\section{Conclusions and Future Perspectives}

This present study showed that the microbiota of the raw milk delivered to the dairy industry continuously shifts in composition, possibly even on a daily basis. We detected a complex milk microbiota with great intragenus and intraspecies diversity, thus implying highly variable sources of contamination at the farm level. The between-farm variation was expected due to differences in the farm environment, but the variation connected to sampling at the same farm and geographical location was unexpected and cannot be explained by available data. Seemingly random variation in milk microbiota is a challenge for the dairy industry, as the quality of dairy products is related to the quality of the raw milk received for processing. The study of the milk microbiota at farm level can also help the dairy industry to identify farms with undesirable microbiota and to work with farmers to identify the contamination sources and reduce or remove unwanted microorganisms from the milk.

\section{ACKNOWLEDGMENTS}

The authors acknowledge the Norwegian dairy cooperative TINE SA (Oslo, Norway) for their assistance during sampling. This work received financial contributions from the Norwegian Foundation for Research Levy on Agricultural Products (Norway) and the Norwegian Agricultural Agreement Research Fund (Norway) (grant no. 244149/E50), the Faculty of Chemistry, Biotechnology and Food Science at the Norwegian University of Life Sciences (Norway) and TINE SA.

\section{REFERENCES}

Bartoszewicz, M., B. M. Hansen, and I. Swiecicka. 2008. The members of the Bacillus cereus group are commonly present contaminants of fresh and heat-treated milk. Food Microbiol. 25:588-596.

Bonizzi, I., J. N. Buffoni, M. Feligini, and G. Enne. 2009. Investigating the relationship between raw milk bacterial composition, as described by intergenic transcribed spacer-PCR fingerprinting, and pasture altitude. J. Appl. Microbiol. 107:1319-1329.

Braem, G., S. De Vliegher, B. Verbist, M. Heyndrickx, F. Leroy, and L. De Vuyst. 2012. Culture-independent exploration of the teat apex microbiota of dairy cows reveals a wide bacterial species diversity. Vet. Microbiol. 157:383-390.

Callahan, B. J., P. J. McMurdie, M. J. Rosen, A. W. Han, A. J. A. Johnson, and S. P. Holmes. 2016. DADA2: High-resolution sample inference from Illumina amplicon data. Nat. Methods 13:581-583.

Cleto, S., S. Matos, L. Kluskens, and M. J. Vieira. 2012. Characterization of contaminants from a sanitized milk processing plant. PLoS One 7:e40189.

Coorevits, A., V. De Jonghe, J. Vandroemme, R. Reekmans, J. Heyrman, W. Messens, P. De Vos, and M. Heyndrickx. 2008. Comparative analysis of the diversity of aerobic spore-forming bacteria in raw milk from organic and conventional dairy farms. Syst. Appl. Microbiol. 31:126-140.

De Jonghe, V., A. Coorevits, K. Van Hoorde, W. Messens, A. Van Landschoot, P. De Vos, and M. Heyndrickx. 2011. Influence of storage vonditions on the growth of Pseudomonas species in refrigerated raw milk. Appl. Environ. Microbiol. 77:460-470.

Delgado, S., C. T. C. C. Rachid, E. Fernandez, T. Rychlik, A. Alegria, R. S. Peixoto, and B. Mayo. 2013. Diversity of thermophilic bacteria in raw, pasteurized and selectively-cultured milk, as assessed by culturing, PCR-DGGE and pyrosequencing. Food Microbiol. $36: 103-111$.

Doyle, C. J., D. Gleeson, P. W. O'Toole, and P. D. Cotter. 2017a. High-throughput metataxonomic characterization of the raw milk microbiota identifies changes reflecting lactation stage and storage conditions. Int. J. Food Microbiol. 255:1-6.

Doyle, C. J., D. Gleeson, P. W. O'Toole, and P. D. Cotter. 2017b. Impacts of seasonal housing and teat preparation on raw milk microbiota: A high-throughput sequencing study. Appl. Environ. Microb. 83: e02694-16. https://doi.org/10.1128/AEM.02694-16.

Edgar, R. C. 2010. Search and clustering orders of magnitude faster than BLAST. Bioinformatics 26:2460-2461.

Elmoslemany, A. M., G. P. Keefe, I. R. Dohoo, J. J. Wichtel, H. Stryhn, and R. T. Dingwell. 2010. The association between bulk tank milk analysis for raw milk quality and on-farm management practices. Prev. Vet. Med. 95:32-40.

Feligini, M., S. Panelli, R. Sacchi, M. Ghitti, and E. Capelli. 2015. Tracing the origin of raw milk from farm by using Automated 
Ribosomal Intergenic Spacer Analysis (ARISA) fingerprinting of microbiota. Food Control 50:51-56.

Fricker, M., B. Skanseng, K. Rudi, B. Stessl, and M. Ehling-Schulz. 2011. Shift from farm to dairy tank milk microbiota revealed by a polyphasic approach is independent from geographical origin. Int. J. Food Microbiol. 145:S24-S30.

Hernandez-Sanabria, E., L. L. Guan, L. A. Goonewardene, M. J. Li, D. F. Mujibi, P. Stothard, S. S. Moore, and M. C. Leon-Quintero. 2010. Correlation of particular bacterial PCR-denaturing gradient gel electrophoresis patterns with bovine ruminal fermentation parameters and feed efficiency traits. Appl. Environ. Microbiol. 76:6338-6350.

Hou, Q., H. Y. Xu, Y. Zheng, X. X. Xi, L. Y. Kwok, Z. H. Sun, H. P. Zhang, and W. Y. Zhang. 2015. Evaluation of bacterial contamination in raw milk, ultra-high temperature milk and infant formula using single molecule, real-time sequencing technology. J. Dairy Sci. 98:8464-8472.

Ivy, R. A., M. L. Ranieri, N. H. Martin, H. C. den Bakker, B. M. Xavier, M. Wiedmann, and K. J. Boor. 2012. Identification and characterization of psychrotolerant sporeformers associated with fluid milk production and processing. Appl. Environ. Microbiol. 78:1853-1864.

Jacobs, J. A., and J. M. Siegford. 2012. Invited review: The impact of automatic milking systems on dairy cow management, behavior, health, and welfare. J. Dairy Sci. 95:2227-2247.

Jago, J. G., K. L. Davis, P. J. Copeman, and M. M. Woolford. 2006. The effect of pre-milking teat-brushing on milk processing time in an automated milking system. J. Dairy Res. 73:187-192.

Kable, M. E., Y. Srisengfa, M. Laird, J. Zaragoza, J. McLeod, J. Heidenreich, and M. L. Marco. 2016. The core and seasonal microbiota of raw bovine milk in tanker trucks and the impact of transfer to a milk processing facility. MBio 7:e00836-16.

Li, N., Y. Z. Wang, C. P. You, J. Ren, W. Y. Chen, H. J. Zheng, and Z. M. Liu. 2018. Variation in raw milk microbiota throughout 12 months and the impact of weather conditions. Sci. Rep. 8:2371.

Lozupone, C., and R. Knight. 2005. UniFrac: A new phylogenetic method for comparing microbial communities. Appl. Environ. Microbiol. 71:8228-8235.

Magnusson, M., A. Christiansson, and B. Svensson. 2007. Bacillus cereus spores during housing of dairy cows: Factors affecting contamination of raw milk. J. Dairy Sci. 90:2745-2754.

Mallet, A., M. Gueguen, F. Kauffmann, C. Chesneau, A. Sesboue, and N. Desmasures. 2012. Quantitative and qualitative microbial analysis of raw milk reveals substantial diversity influenced by herd management practices. Int. Dairy J. 27:13-21.

Marchand, S., J. De Block, V. De Jonghe, A. Coorevits, M. Heyndrickx, and L. Herman. 2012. Biofilm formation in milk production and processing environments; influence on milk quality and safety. Compr. Rev. Food Sci. Food Saf. 11:133-147.

Masiello, S. N., N. H. Martin, R. D. Watters, D. M. Galton, Y. H. Schukken, M. Wiedmann, and K. J. Boor. 2014. Identification of dairy farm management practices associated with the presence of psychrotolerant sporeformers in bulk tank milk. J. Dairy Sci. 97:4083-4096.

Oikonomou, G., M. L. Bicalho, E. Meira, R. E. Rossi, C. Foditsch, V. S. Machado, A. G. V. Teixeira, C. Santisteban, Y. H. Schukken, and R. C. Bicalho. 2014. Microbiota of cow's milk; distinguishing healthy, sub-clinically and clinically diseased quarters. PLoS One 9:e85904.

Osterås, O., L. Solverod, and O. Reksen. 2006. Milk culture results in a large Norwegian survey-effects of season, parity, days in milk, resistance, and clustering. J. Dairy Sci. 89:1010-1023.

Paulson, J. N., O. C. Stine, H. C. Bravo, and M. Pop. 2013. Differential abundance analysis for microbial marker-gene surveys. Nat. Methods 10:1200-1202.

Porcellato, D., M. Aspholm, S. B. Skeie, M. Monshaugen, J. Brendehaug, and H. Mellegard. 2018. Microbial diversity of consump- tion milk during processing and storage. Int. J. Food Microbiol. 266:21-30.

Porcellato, D., and S. B. Skeie. 2016. Bacterial dynamics and functional analysis of microbial metagenomes during ripening of Dutchtype cheese. Int. Dairy J. 61:182-188.

Quigley, L., R. McCarthy, O. O'Sullivan, T. P. Beresford, G. F. Fitzgerald, R. P. Ross, C. Stanton, and P. D. Cotter. 2013a. The microbial content of raw and pasteurized cow milk as determined by molecular approaches. J. Dairy Sci. 96:4928-4937.

Quigley, L., O. O'Sullivan, T. P. Beresford, R. P. Ross, G. F. Fitzgerald, and P. D. Cotter. 2011. Molecular approaches to analysing the microbial composition of raw milk and raw milk cheese. Int. J. Food Microbiol. 150:81-94.

Quigley, L., O. O'Sullivan, C. Stanton, T. P. Beresford, R. P. Ross, G. F. Fitzgerald, and P. D. Cotter. 2013b. The complex microbiota of raw milk. FEMS Microbiol. Rev. 37:664-698.

R Core Team. 2017. R: A language and environment for statistical computing. R Foundation for Statistical Computing, Vienna, Austria. https://www.R-project.org/.

Reksen, O., L. Solverod, A. J. Branscum, and O. Osteras. 2006. Relationships between milk culture results and treatment for clinical mastitis or culling in Norwegian dairy cattle. J. Dairy Sci. 89:2928-2937.

Rodrigues, M. X., S. F. Lima, S. G. Canniatti-Brazaca, and R. C. Bicalho. 2017. The microbiome of bulk tank milk: Characterization and associations with somatic cell count and bacterial count. J. Dairy Sci. 100:2536-2552.

Salustiano, V. C., N. J. Andrade, N. F. F. Soares, J. C. Lima, P. C. Bernardes, L. M. P. Luiz, and P. E. Fernandes. 2009. Contamination of milk with Bacillus cereus by post-pasteurization surface exposure as evaluated by automated ribotyping. Food Control 20:439-442.

Statistics Norway. 2017. Livestock husbandry. Accessed April 2018. https://www.ssb.no/en/jord-skog-jakt-og-fiskeri/statistikker/ jordhus/aar.

Tenhagen, B. A., G. Koster, Wallmann, and J. W. Heuwieser. 2006. Prevalence of mastitis pathogens and their resistance against antimicrobial agents in dairy cows in Brandenburg, Germany. J. Dairy Sci. 89:2542-2551.

Vacheyrou, M., A. C. Normand, P. Guyot, C. Cassagne, R. Piarroux, and Y. Bouton. 2011. Cultivable microbial communities in raw cow milk and potential transfers from stables of sixteen French farms. Int. J. Food Microbiol. 146:253-262.

Vaerewijck, M. J. M., P. De Vos, L. Lebbe, P. Scheldeman, B. Hoste, and M. Heyndrickx. 2001. Occurrence of Bacillus sporothermodurans and other aerobic spore-forming species in feed concentrate for dairy cattle. J. Appl. Microbiol. 91:1074-1084.

Vithanage, N. R., M. Dissanayake, G. Bolge, E. A. Palombo, T. R. Yeager, and N. Datta. 2016. Biodiversity of culturable psychrotrophic microbiota in raw milk attributable to refrigeration conditions, seasonality and their spoilage potential. Int. Dairy J. $57: 80-90$.

von Neubeck, M., C. Baur, M. Krewinkel, M. Stoeckel, B. Kranz, T. Stressler, L. Fischer, J. Hinrichs, S. Scherer, and M. Wenning. 2015. Biodiversity of refrigerated raw milk microbiota and their enzymatic spoilage potential. Int. J. Food Microbiol. 211:57-65.

Waage, S., T. Mork, A. Roros, D. Aasland, A. Hunshamar, and S. A. Odegaard. 1999. Bacteria associated with clinical mastitis in dairy heifers. J. Dairy Sci. 82:712-719.

Xia, Y. L., and J. Sun. 2017. Hypothesis testing and statistical analysis of microbiome. Genes Dis. 4:138-148.

Young, W., B. C. Hine, O. A. M. Wallace, M. Callaghan, and R. Bibiloni. 2015. Transfer of intestinal bacterial components to mammary secretions in the cow. PeerJ 3:e888.

Zadoks, R. N., R. N. Gonzalez, K. J. Boor, and Y. H. Schukken. 2004. Mastitis-causing streptococci are important contributors to bacterial counts in raw bulk tank milk. J. Food Prot. 67:2644-2650. 